\title{
What does the Comparative Method Reveal About Adaptation?
}

\section{Citation}

Leroi, A. M., M. R. Rose, and G. V. Lauder. 1994. “What Does the Comparative Method Reveal About Adaptation?" The American Naturalist 143 (3) (March): 381-402. doi:10.1086/285609.

\section{Published Version}

doi:10.1086/285609

\section{Permanent link}

http://nrs.harvard.edu/urn-3:HUL.InstRepos:33948436

\section{Terms of Use}

This article was downloaded from Harvard University's DASH repository, and is made available under the terms and conditions applicable to Other Posted Material, as set forth at http:// nrs.harvard.edu/urn-3:HUL.InstRepos:dash.current.terms-of-use\#LAA

\section{Share Your Story}

The Harvard community has made this article openly available.

Please share how this access benefits you. Submit a story.

Accessibility 


\title{
WHAT DOES THE COMPARATIVE METHOD REVEAL ABOUT ADAPTATION?
}

\author{
A. M. Leroi, M. R. Rose, and G. V. Lauder* \\ Department of Ecology and Evolutionary Biology, University of California, Irvine, California 92717
}

Submitted July 6, 1992; Revised March 4, 1993; Accepted April 27, 1993

\begin{abstract}
It has been suggested recently that new quantitative methods for analyzing comparative data permit the identification of evolutionary processes. Specifically, it has been proposed that new comparative methods can distinguish the direct effects of natural selection on the distribution of a trait within a clade from the effects of drift, indirect selection, genotype-byenvironment interaction, and uncontrolled environmental variation. Such methods can supposedly unravel the relative importance of these factors by the phylogenetic analysis of traits, performance attributes, and habitats. We argue that they cannot. We show that many different evolutionary mechanisms can, in principle, account for any one interspecific pattern, and we illustrate our case using examples from the comparative literature. We argue that these confounded mechanisms can only be unraveled if patterns of selection or genetic variation and covariation are directly measured in many species within a clade. Even though comparative methods are valuable for examining the evolutionary history of traits, they will often mislead in the study of adaptive precesses.
\end{abstract}

The use of comparisons among extant species to identify adaptations has many contemporary practitioners, as well as a pedigree stretching back to the Origin of Species, if not to Paley's theistic biology (Darwin 1859, 1871; Simpson 1953; Bock 1977; Clutton-Brock and Harvey 1979; Gould and Lewontin 1979; Trivers 1985; Coddington 1988; Baum and Larson 1991; Brooks and McLennan 1991; Ridley 1992). More recently, the comparative method has also been used to identify traits that are apparently not adaptive and the constraints that cause them to be so (Coddington 1988; Baum and Larson 1991; Brooks and McLennan 1991). That is, comparative methods are now being used to reveal the mechanisms of evolution. This article examines the foundation of this research strategy.

First, we must define the scope of our inquiry. What is meant by "the mechanisms of evolution," and what kinds of studies make claims about them? By mechanisms of evolution we mean all those forces, such as genetic drift, natural selection, mutation, or migration, that mold the evolution of populations or clades. We think that claims about such mechanisms are made more often than is readily apparent, for instance, whenever a trait is identified as an adaptation. This position may seem peculiar at first. After all, a distinction is often drawn between adaptation as a process and adaptations as traits (Futuyma 1986). But, as many discussions of adaptation and adaptations show, this distinction tends

\footnotetext{
* To whom correspondence should be addressed. E-mail: glauder@vmsa.oac.uci.edu.
} 
to dissolve as these terms are more rigorously defined. Sober (1984, p. 208), for example, characterizes an adaptation as follows: " $A$ is an adaptation for task $T$ in population $P$ if and only if $A$ became prevalent in $P$ because there was selection for $A$, where the selective advantage of $A$ was due to the fact that $A$ helped perform task T." By Sober's definition, adaptations (as states) are, in part, defined by the evolutionary process-direct selection for those traits-that gave rise to them.

Some definitions of adaptation distinguish between the causes of the historical genesis of a trait and its current maintenance by selection: "we may designate as an adaptation any feature that promotes fitness and was built by selection for its current role" (Gould and Vrba, 1982, p. 6). This is a more historical view of adaptation than Sober's. As such, it lends itself naturally to a comparative or phylogenetic context, and as the analysis of interspecific patterns has become more sophisticated over recent years, it has been widely adopted. Coddington (1988, p. 3), for example, defines an adaptation as an "apomorphic function promoted by natural selection" and states elsewhere that "an evolutionary definition of adaptation must have an historical component specifying selection as the evolutionary agent responsible for the appearance of the feature"' (p. 5). In their definition of an adaptation, Baum and Larson (1991) add to this by explicitly incorporating the idea of a trait's performance; stated in a way comparable to Sober's, it might read as follows: $A^{\prime}$ is an adaptation for task $T$ in clade $C$ if and only if (1) $A^{\prime}$ is currently maintained by natural selection in $C$ because of its superior performance $\alpha^{\prime}$ at task $T$ relative to the ancestral condition $A$ and its performance $\alpha$, and (2) $A^{\prime}$ originally became prevalent in $C$ because of selection for its superior performance $\alpha^{\prime}$ at task T. Baum and Larson (1991) define maladaptive and selectively neutral traits in an analogous fashion: if the performance of the derived trait is lower than that of the ancestral condition $\left(\alpha^{\prime}<\alpha\right)$, then $A^{\prime}$ is a "disaptation"; if the derived performance is equivalent in in-group and out-group taxa $\left(\alpha^{\prime}=\alpha\right)$, then $A^{\prime}$ is a "nonaptation." The important point is that, like Sober's, all these definitions of adaptations are founded on statements about an evolutionary process: natural selection.

Given that adaptations can be defined in principle, how should they be identified in practice? Most biologists would agree that, at a minimum, the function of a putative adaptation should be well understood. Many others would add that the phylogenetic distribution of a trait should also be known. Baum and Larson (1991) attempt to apply their definition. They argue that identification of the phylogenetic distribution of a derived trait, its performance relative to the ancestral trait, and the "selective regime" that putatively influences its evolution are necessary and sufficient to distinguish between adaptive and nonadaptive traits. This position, or one similar to it, is held by Greene (1986) and Coddington (1988), as well as Harvey and Pagel (1991, p. 11), who assert, "Stripped to the bone, . . . the evidence for adaptive evolution revealed by comparative studies is correlated evolution among characters and environments." It is an attractive view, for it offers the hope that clades can be inventoried for adaptations, nonaptations, and disaptations without having actually to measure natural selection operating on traits. 
In this article, we take issue with this position. We will argue that knowledge of the phylogenetic distribution of traits, their performance, and selective regimes are not sufficient to identify adaptations. This is not a completely novel perspective; both Mishler (1988) and Brandon (1990) have stated that a complete adaptive explanation requires both a demonstration of the relative fitness of a putative adaptation and the trait it supplants, as well as genetic information. We agree. But we will go further than these authors and argue that, in the absence of such broader information, phylogenetic patterns will often suggest that a trait is an adaptation when in fact it is not and suggest that it is not, when in fact it is.

We begin our argument with a discussion of theoretical and empirical findings from population genetics and show how the effects of selection, patterns of genetic covariation, genetic background, environment, and the interaction of genotypes with their environment influence the observed distribution of traits among populations, species, and higher taxa. Given these factors, we reevaluate recent articles-both methodological and specific case studies-that claim to infer adaptation by the comparative method. Finally, we consider the general implications of our critique, particularly with respect to the circumstances in which the comparative method can legitimately be used to analyze adaptation and evolutionary constraint.

\section{POPULATION GENETICS OF MULTIPLE FITNESS-RELATED CHARACTERS}

It is our primary intent in this section to show how any single comparative pattern can arise by means of very different evolutionary genetic mechanisms. Formally, our basic arguments can be developed in terms of a two-character, two-allele, one-locus model, though later we consider other loci and characters in the development of our argument. Say that we have two alleles at a locus, $B_{1}$ and $B_{2}$, that can affect two distinct characters, $C_{1}$ and $C_{2}$, with any pattern of dominance:

Character \begin{tabular}{cccc}
\multicolumn{3}{c}{ Genotype } \\
& $B_{1} B_{1}$ & $B_{1} B_{2}$ & $B_{2} B_{2}$ \\
$C_{1}$ & $H_{1}+\beta_{1}$ & $H_{1}$ & $H_{1}+\beta_{2}$ \\
$C_{2}$ & $H_{2}+\gamma_{1}$ & $H_{2}$ & $H_{2}+\gamma_{2}$
\end{tabular}

The $\beta_{i}$ and $\gamma_{i}$ specify the allelic effects and may have any sign; $H_{i}$ specifies the phenotypic effect of the genetic background and may also have any sign. Some trivial points follow immediately. If the $\beta_{i}$ are both zero and the $B$ locus is the only one affecting $C_{1}$, then there will be no genetic variation for $C_{1}$ even when this locus is polymorphic. In quantitative genetic notation, $V_{\mathrm{G}}\left(C_{1}\right)=0$ when all $\beta_{i}=0$. Similar conclusions follow for $C_{2}$. However, if at least one $\beta_{i}$ and at least one $\gamma_{i}$ are different from zero, then $V_{G}\left(C_{1}\right)>0$ and $V_{G}\left(C_{2}\right)>0$ when there is genetic polymorphism. Of greater interest, there will be a genetic correlation between characters $C_{1}$ and $C_{2}$ when there is segregating genetic variation affecting both at locus $B$. The particular values for the genetic variances and correlation will depend on the gene frequencies of $B_{1}$ and $B_{2}$ (since the total gene frequency 
must equal one, there is only one gene frequency to keep track of in a two-allele system). Let $p$ designate the allele frequency of $B_{1}$, and assume that there is random mating with respect to the genotypes at this locus. In this model, the values of the characters $C_{1}$ and $C_{2}$ in any species are determined by the values of $H_{i}, \beta_{i}, \gamma_{i}$, and $p$. The distribution of $C_{1}$ and $C_{2}$ across a set of populations or species will, likewise, depend on the distributions of these variables. We discuss the influence of these variables on interspecific patterns next.

\section{Interspecific Patterns Due to Pleiotropy, Epigenetics, and Correlated Selection}

Consider one of the simplest possible patterns of interspecific association of $C_{1}$ and $C_{2}$ : a positive one. How might this be explained? At least three elementary hypotheses, of a sort common in the comparative literature, present themselves; we call these the pleiotropy, the epigenetic, and the correlated selection hypotheses. The pleiotropy hypothesis is so called because it invokes, on the basis of a presumed functional connection between $C_{1}$ and $C_{2}$, a positive genetic covariance between these traits, arising from pleiotropy. In this scenario, selection acts on one of these characters alone. In terms of the previously cited model, the allelic effects might be $\beta_{2}>\beta_{1}$ and $\gamma_{2}>\gamma_{1}$, and fitness might be a linear function of $C_{2}$ but have no dependence on $C_{1}$. A range of species with varying selection histories would differ in $p$ : some would be fixed for $B_{1}$, some fixed for $B_{2}$, and some would be polymorphic. This condition would give rise to an interspecific association among $C_{1}, C_{2}$, and fitness.

The epigenetic hypothesis is similar to the pleiotropy hypothesis in that the interspecific correlation between $C_{1}$ and $C_{2}$ is a consequence of selection on one of these traits and the correlated evolution of the other. Here, however, the functional connection-which is a consequence of development-gives rise to a complex kind of pleiotropy: an epigenetic interaction (sensu Atchley and Hall 1991). An epigenetic interaction might occur if, say, $C_{1}$ were to influence the development of $C_{2}$, perhaps through an inductive event, but $C_{2}$ were to have no influence on the development of $C_{1}$. This means that most genes that affect $C_{1}$ would also affect $C_{2}: \beta_{i}$ not equal to zero would necessarily imply $\gamma_{i}$ not equal to zero and vice versa. But for genes that affect $C_{2}, \gamma_{i}$ not equal to zero implies nothing about $\beta_{i}$. This functional asymmetry gives rise to an asymmetry in genetic correlations: $C_{2}$ would be genetically correlated with $C_{1}$ when the latter has genetic polymorphism, but the reverse would not be necessarily true (Atchley and Hall 1991). This situation, in turn, could give rise to an asymmetry in selection responses depending on the genetic effects and the characters undergoing selection.

The correlated selection hypothesis in its simplest form postulates that fitness is a linear function of traits $C_{1}$ and $C_{2}$ in a given environment, but $C_{1}$ and $C_{2}$ are not associated by either phenotypic or genetic correlations. Here, locus $B$ might influence character $C_{1}$ but not $C_{2}$; that is, the $\beta_{i}$ are not equal to zero, but the $\gamma_{i}$ are equal to zero, while alleles influencing only $C_{2}$ segregate at other loci. For instance, the activity of a glycolytic enzyme and a biomechanical trait such as stride length, in both contributing to maximal running speed, might be selected 
upward together under conditions that select for running speed. In such a case, selection will, over a range of species, also give rise to a positive interspecific correlation between $C_{1}$ and $C_{2}$ and fitness, despite an absence of genetic correlation.

The pleiotropy, epigenetic, and correlated selection hypotheses differ in their assumptions about the genetic and phenotypic covariance structure of the evolving species, as well as differing in their assumptions about the historical forces of selection acting on each trait. Comparative data offer no hope of discriminating among these evolutionary genetic hypotheses for the simple reason that nothing can be inferred about patterns of phenotypic and genetic correlations within populations from population (or species) means. Functional analysis of the characters will also be generally unhelpful, for a mechanical association between two traits may be reflected in either or both of the genetic or the phenotypic correlation structure of the characters. Conversely, patterns of genetic and phenotypic covariance need not be dependent on obvious mechanical associations. These hypotheses are but the most elementary of an enormous range of evolutionary genetic mechanisms that can give rise to any given interspecific pattern of association.

\section{Interspecific Patterns Due to Environmental Covariance}

We can extend this model to include phenotypic responses to environmental variation of each character:

$$
\begin{array}{cccc} 
& B_{1} B_{1} & B_{1} B_{2} & B_{2} B_{2} \\
\text { Character } & H_{1}+\beta_{1}+\delta(E) & H_{1}+\delta(E) & H_{1}+\beta_{2}+\delta(E) \\
C_{2} & H_{1}+\gamma_{1}+\epsilon(E) & H_{2}+\epsilon(E) & H_{2}+\gamma_{2}+\epsilon(E)
\end{array}
$$

The $\delta$ and $\epsilon$ specify the response of each character to environmental variation $(E)$. If the $\delta$ and $\epsilon$ terms are of the same sign over the range of environments, then $C_{1}$ and $C_{2}$ respond to environmental variation in a similar way; that is, they have a positive environmental covariance. In the absence of genetic covariance $\left(\beta_{i}=\gamma_{i}=0\right.$ at all loci), $\delta$ and $\epsilon$ will determine the sign of the phenotypic covariance between $C_{1}$ and $C_{2}$. Environmental covariances, like genetic covariances, may reflect functional connections between traits. The extent to which this is important in the comparative pattern of multiple traits depends on the extent to which environmental variation is allowed to obscure the genetic differences among populations; if all populations compared are reared in a common environment, then this criticism has no force. Comparative data are, however, often based on organisms reared under very different environmental conditions. When this is so, inferences about evolutionary processes become dependent on physiological homeostasis in a way that is unpredictable in the absence of extensive knowledge of the comparative physiology of the species concerned.

\section{Interspecific Patterns Due to Drift and Linkage}

Even if neither of the observed characters is related to fitness (either by genetic effect or by statistical association with other allele frequencies or fitness charac- 
ters), an interspecific correlation could still arise due to genetic drift. If $C_{1}$ and $C_{2}$ are the only characters affected by locus $B$, and alleles $B_{1}$ and $B_{2}$ are neutral, $p$ will be determined by mutation pressure and genetic drift (see Lande 1979; Kimura 1983). Such purely neutral evolution is unlikely to generate consistent comparative patterns for any single character or pair of characters. However, if many characters are surveyed, then there is the possibility of neutral evolution generating a spuriously significant comparative association, when proper allowance for false positives is not made (Felsenstein 1985). Of course, such characters might still be useful for reconstructing phylogenetic patterns among taxa.

It may also be the case that $C_{1}$ and $C_{2}$ are not fitness-related characters but have accidental associations with other fitness-related characters, perhaps because of linkage disequilibrium, as reflected in a transient genetic correlation with each other and fitness components. In these cases, the values of $p, C_{1}$, and $C_{2}$ will be determined by accidents of sampling in the evolution of each population. Unless locus $B$ (and similarly for all other loci affecting the two characters) is extremely close to a locus under selection or part of an inversion containing other loci under selection, then the evolution of $C_{1}$ and $C_{2}$ over a range of disparate species should not follow any discernible pattern. In the case of the Adh locus in Drosophila melanogaster, in which patterns of linkage have been studied intensively, linkage disequilibrium decays rapidly within the locus (Kreitman 1983), which suggests that physical proximity of loci is not likely to be a factor in the evolution of characters unrelated to fitness, at least for species with effective population sizes comparable to those of $D$. melanogaster. However, evolutionary units such as mitochondria, inversions, and small chromosomes with suppressed recombination all might generate historical associations over groups of species in which these units are preserved. It is an open question how important such associations are in the groups of species normally used in comparative research.

\section{Interspecific Patterns Due to Evolution of the Genetic Background}

Species may vary in their genetic backgrounds because of a number of causes and do so in such a way as to obscure or even alter the genetic relations among traits. Since selection for either character will tend to alter the other as a correlated response, it may be expected that the correlation between these two characters would be preserved among species. But this need not be so. Consider, for example, a case of antagonistic pleiotropy (see Rose 1982), in which $\beta_{1}>0>\gamma_{1}$ and $\gamma_{2}>0>\beta_{2}$. Let fitness be an increasing function of $C_{1}$ and $C_{2}$, of some form (Rose 1982). With these genetic parameters, it will often be the case that overdominance arises, and thus does protected polymorphism (Rose 1982, 1983). Moreover, at polymorphic equilibria, the additive genetic correlation between the two characters will be -1 . In other words, this is a simple case of an evolutionary trade-off between two fitness-related characters.

One potential problem is other loci for which the signs of the $\beta_{i}$ and $\gamma_{i}$ are the same. Houle (1991) has considered cases of this kind, in which $C_{1}$ and $C_{2}$ are the final products of a metabolic pathway that branches just before the characters. Thus, there are allocative effects on the relative balance between the two characters at the branching point that could give rise to genetic effects like those that 
arise when the $\beta_{i}$ and $\gamma_{i}$ are of opposite signs, and acquisitional effects enhancing both characters, which would give rise to loci with genetic effects that have the same sign. While the latter type of locus would not have genetic polymorphism sustained by selection, the fixation of different beneficial alleles of this type among different species would result in genetic background parameters that would positively co-vary. If the magnitude of the variance generated between species by such substitutions is sufficiently greater than that generated between species by different values for $p$ at allocative loci, then the existence of a functional trade-off, and an associated negative genetic correlation among species, between the two characters could be wholly obscured.

Species may differ in genetic background because of one of at least three causes. First, the amount and kind of genetic variation may differ among species such that identical ecological pressures will give rise to different evolutionary responses. With respect to the previously mentioned case, it could be that, even though increased acquisition is strongly selected for in all species, only some species have the genetic variation required for an evolutionary response. Second, even though species may have identical classes of mutations available to them, subtle differences in ecological conditions can cause gene frequencies to converge on different equilibria. Species selected just for varying degrees of acquisition will not show any trade-off in how they allocate their resources. Third (though this is probably the least important cause in nature), species may differ in the degree to which they experience inbreeding depression. If the number of acquisitive loci is larger than the number of allocative loci, then the fixation of deleterious alleles with inbreeding should tend to result in a positive covariation for functional characters, in the same way that inbreeding laboratory lines will (Rose 1984a). Variation in the overall degree of inbreeding depression can be due to variation in historical effective population sizes $\left(N_{\mathrm{e}}\right)$ or, if these are similar among species, the stochastic fixation of different deleterious alleles at different loci in separate evolutionary lineages.

This confounding influence of genetic background on interspecific comparisons is not restricted to characters that are the end points of branched metabolic pathways. It arises whenever characters are influenced by multiple loci, associated by complex patterns of pleiotropy, and under complex patterns of selection. We believe that these conditions will be met for many of the traits that are commonly the subject of comparative analysis and that it is merely the absence of information about the genetic basis of such traits in most organisms that permits comparative biologists to ignore the vagaries of genetic structure in their inferences of evolutionary mechanism via comparative analyses.

Much, however, is known about the genetic basis of fitness-related traits in at least one species, Drosophila melanogaster. Quantitative genetic studies have revealed over the past decade that patterns of pleiotropy are pervasive and unpredictable among characters at all levels of organismal analysis. For example, replicated strains selected for late-life fecundity in various laboratories exhibit correlated increases in longevity and flight endurance (Rose 1984b; Luckinbill et al. 1988) as well as stress resistance traits such as ethanol, desiccation, and starvation resistance (Service et al. 1985, 1988). These same strains show declines in 
early-life fecundity (Rose 1984b), early-life male competitive mating ability (Service 1993), and early-life metabolic rate (Service 1987). The increases in stress resistance and flight endurance are, in turn, associated with increases in lipid and glycogen metabolite pool sizes (Service 1987; Graves et al. 1992).

If, as these studies suggest, pleiotropy is common in large outbred populations, then the comparative approach is weakened as a method for inferring adaptive mechanisms. For not only does it become very difficult to attribute interspecific evolution in any given trait to selection for that trait (rather than for another with which it is genetically correlated), but, as we argued for the case of a $Y$-shaped metabolic pathway, selection at one trait can obscure the genetic relationships that exist between other traits when viewed across species. Finally, the possibility exists that the genetic background might evolve in such a way so as not merely to obscure the genetic relationship between traits within any given population but actually to alter them.

Since genetic correlations are dependent on allele frequencies as well as patterns of allelic effects, selection (or drift) can, in principle, cause them to be highly unstable in the course of evolution. The degree to which they are unstable has been the center of theoretical disagreement for some time (Turelli 1988; Slatkin and Frank 1990), and evidence bearing on their stability is scattered and weak. Genetic correlations have occasionally been found to evolve in laboratory populations. In the course of subjecting Drosophila populations to two-trait selection, Sheridan and Barker (1974) demonstrated that genetic correlations between coxal and sternopleural bristles may diverge, though not necessarily in a manner congruent with theoretical expectations. Genetic correlations have also been found to respond to one-trait selection in Tribolium and Drosophila populations (see Wilkinson et al. 1990). Much evidence for the lability of genetic correlations in natural populations is ambiguous (see, e.g., Arnold 1981; Lofsvold 1986; Kohn and Atchley 1988; Cowley and Atchley 1990; other references in Barton and Turelli 1989), but there is at least one demonstration of evolved -differences in genetic covariance structure (Atchley et al. 1992). The intensity of directional selection required to change the signs and magnitudes of genetic correlations in the laboratory is not beyond the range of those found in nature (Endler 1986).

The possibility exists, then, that such correlations change frequently in the course of the divergence of sister lineages, in which case a comparison of the mean phenotype of such lineages will reveal nothing of the genetic relations among traits that exist within each particular lineage, at any one time.

\section{Genotype-by-Environment Interaction and the Genetic Background}

The confounding effect of genetic background becomes even more acute when interactions between specific alleles and genetic backgrounds exist or when specific genetic backgrounds interact with environment. The first of these types of interaction, epistasis, is generally thought to be less pervasive than simple additive background effects. Yet from his studies of gene action in guinea pig coloration and morphology, Wright (1977) believed that epistasis was important in the evolution of many traits, and it occupied a central place in his shifting balance theory of evolution. Modern metabolic control theory has confirmed his view 
that epistasis is a systemic property of multistep metabolic pathways (Keightley 1989). Epistatic effects may cause the effects (the $\beta_{i}$ and $\gamma_{i}$ ) of particular alleles to alter, taking, for instance, positive values on some genetic backgrounds but negative values on others, and so to alter genetic correlations.

While epistatic interactions are commonly found among mutations of large effect on arbitrary traits (Wright 1977) or in crosses of inbred lines (see, e.g., Jinks et al. 1973; Clark and Feldman 1981; Clark et al. 1981; Seager and Ayala 1982), far less is known about their influence on fitness-related traits in outbred populations (Crow 1987). In a few cases, however, epistatic modifiers have been shown to alter patterns of pleiotropy in laboratory populations. Clarke and McKenzie (1987) showed that alleles that confer resistance to the insecticide diazinon in the blowfly, Lucilia cuprina, have the pleiotropic effect of increasing developmental instability (as measured by fluctuating asymmetry). These pleiotropic effects are not, however, reflected in negative correlations between Diazinon resistance and these fitness-related traits across populations; rather, Diazinon-resistant populations have compensated in some fashion for the deleterious pleiotropic effects of resistance (McKenzie et al. 1982; Clarke and McKenzie 1987). This compensation was shown to be due to the particular composition of the genetic background of Diazinon-resistant flies-that is, due to evolved epistatic modifiers. In a similar case, Lenski $(1988 a, 1988 b)$ showed that mutations in Escherichia coli that confer resistance to the virus $\mathrm{T} 4$ have deleterious pleiotropic effects on competitive fitness and that these effects could be modified by subsequent evolution in the absence of T4. Remarkably, the increase in fitness in the resistant strains was not attributable to a reversion at the resistance locus but rather to the evolution of an allele elsewhere in the genome that epistatically diminished the cost of resistance.

It is well-known that the expression of genetic relations (such as pleiotropy) can vary among environments because of interactions between the environment and the collection of genotypes segregating within populations (Service and Rose 1985; de Jong 1990; Stearns et al. 1991). Much the same phenomenon can arise owing to interactions between the environment and the differentiated genetic backgrounds of a collection of species or populations. Indeed, many instances are known of related populations having different patterns of environmental sensitivity or "reaction norms" (see, e.g., Berven and Gill 1982; Schlichting and Levin 1986). In this case, species that have similar evolutionary genetic mechanisms at work in their normal environments could exhibit comparative patterns in a common assay environment that do not reflect these mechanisms. In particular, species can be expected to differ with respect to their functional attributes under novel or stressful conditions (Hoffmann and Parsons 1991) and so may be expected to exhibit positive interspecific covariation for such traits in some artificial or laboratory environments.

\section{The Necessity of Skepticism}

Despite this list of ways in which various evolutionary genetic mechanisms can be confounded with each other, there are, no doubt, cases in which the pattern of interspecific differentiation does reflect a simple evolutionary genetic mecha- 
nism. For example, Arnold (1981) found that the chemoreceptive response to slug and leech odor was positively associated between an inland and a coastal population of garter snakes. His explanation of this association was of the sort that we have called the pleiotropy hypothesis: a genetic correlation (in this case positive) combined with divergent selection (for chemoreception of slug odor in the coastal population and against chemoreception of leech odor in the inland population). What makes Arnold's scenario more persuasive than most of this sort is the demonstration that the genetic correlation between these two traits is strongly positive and identical in each of the populations. Even here caution is required, however, for the population parameters were estimated in a common laboratory environment and are confounded with differences in maternal environment. The influence of this novel environment on the population means and genetic correlations is completely unknown. Nevertheless, in providing the information required to weaken the most elementary competing hypothesis (e.g., correlated selection), this study suggests a route out of the thicket of confounding mechanisms.

Very little evidence bears on the evolutionary genetic mechanisms that are responsible for distributions of traits among species, so it is difficult to say which of the mechanisms discussed earlier are most likely to be confounded in any given study. We do not intend here to make a claim for the importance of any one pattern of selection, allelic effects, or genetic structure over another. We claim only the necessity of a healthy skepticism when evolutionary scenarios are offered to explain comparative patterns in the absence of direct evidence about historical selection forces and the genetic relations among traits (see also Cracraft 1981).

\section{DUBIOUS INFERENCES FROM COMPARATIVE DATA}

In this section we illustrate the preceding population genetics arguments using examples of actual comparative studies. We document three classes of erroneous inference based on comparative data and the historical definition of adaptation.

\section{Unwarranted Inferences of Adaptation from Correlations between Characters and Environments}

Ecomorphological correlations have long been used as evidence for the adaptive nature of organismal form (see, e.g., Cody and Diamond 1975; Bock 1977; Luke 1986; Brooks and McLennan 1991). Under the best possible situation, the repeated (phylogenetically independent) occurrence of particular morphological features congruently with a specific environmental factor may provide evidence of a causal relationship between environment and morphology-if the correlation is high and if the environmental factor is precisely identified. One such study (cited as "exemplary" in Mayr 1983) is Traub's (1980) demonstration that a particular pattern of bristle morphology in fleas (the "crown of thorns" phenotype) has evolved independently by different developmental routes in a number of lineages. This trait, thought to assist fleas in attachment to the host, is usually found in species that parasitize hosts that live in habitats especially hazardous to 
fleas and in which there is presumably a selective premium for staying on the host. Another broadly comparative study is that of Luke (1986), who showed that lizard toe fringes have evolved at least 26 times in seven families. Fringe morphology is generally correlated with the type of substrate, although several different types of toe fringes may all be found in species inhabiting a common environment. However, it is possible to argue that some type of toe projection may be adaptive during locomotion in sand, without specifying the exact morphology of the projection.

In fact, few analyses in the literature fit these best-case situations. Often the correlation that is presented is between a morphological trait and a general environmental condition that has originated but once in phylogeny. The example of the plethodontid salamanders discussed by Baum and Larson (1991) as the primary example of their methodology for analyzing adaptations (see also Larson et al. 1981; Larson 1984; Wake and Larson 1987) illustrates these difficulties. The morphological novelty of altered tarsal structure in the genus Aneides is considered to be an adaptation conferring enhanced climbing ability relative to the ancestral condition and is correlated with the origin of an arboreal/scansorial habit. The correlation is, however, based on only two data points: both the morphological novelty and change in habit arose once at the same internode interval on the phylogeny. This type of single historical event involving a change in both morphology and environment is one that has very low power in historial analysis (Lauder 1981): given a single event, a virtually limitless number of scenarios may be outlined that fit the observed datum. Fortunately, this problem has been addressed in at least some of the recent comparative publications (see, e.g., Harvey and Pagel 1991).

A second difficulty with such comparative and historical methods of analyzing adaptation is that often the environment is vaguely treated. The notion of a selective regime (Baum and Larson 1991) or an "adaptive zone" (Simpson 1953; Van Valen 1971; Larson et al. 1981) is so general as to be useless in attempting to identify selection forces that have acted on morphology. Experimental population biology has repeatedly demonstrated the sensitivity of phenotypic responses to small changes in the nature of the imposed selective regime (see, e.g., King 1955). To use a selective regime such as "arboreal/scansorial habit" as a character for the purpose of discerning adaptation combines so many distinct biophysical features of the environment into a single label that comparative tests using such a character are likely to have low resolving power.

\section{The Invocation of Undemonstrated Constraints on Adaptation in the Explanation of Phylogenetic Conservatism}

Gould and Lewontin (1979) set comparative biology the task of elucidating "phyletic" and "developmental" constraints on adaptation, and not one of the recent expositions of the comparative method is without a discussion of such constraints (Coddington 1988; McLennan et al. 1988; Baum and Larson 1991; Brooks and McLennan 1991; Harvey and Pagel 1991). Constraints arguments are especially evident in explanations of phylogenetic conservatism. Coddington (1988, pp. 18-19), for example, states that, "although several exactly specifiable 
convolutions of the ejaculatory duct within the male secondary genitalia of the theridiosomatid spiders defines the family . . . , it is unlikely that anyone would assert that this extraordinary conservatism is caused by relentless stabilizing selection, on the route of the duct in all theriosomatid lineages." He continues, "The darwinian paradigm is best tested at low taxonomic levels. At such levels, true novelties may still be maintained by stabilizing selection in all lineages. Their conversion into developmental 'constraint' and phylogenetic 'inertia' may not have yet happened." In a similar fashion, Brooks and McLennan (1991, p. 166) suggest that the presence of a male-territorial, polygynous, paternal-care mating system in all species of gasterosteid fish is a "reflection of tight phylogenetic constraints on mating-system evolution within this family," offering as evidence against an adaptive explanation the observation that the Gasterosteidae live in a wide range of habitats.

Doubts have recently been raised concerning the coherence of notions such as phylogenetic, developmental, and genetic constraints (Antonovics and van Tienderen 1991; but see Arnold 1992). Those who invoke them to explain phylogenetic conservatism seem to believe one or more of the following. First, many traits may lack sufficient genetic variation for selection, stabilizing or otherwise, to work on. This may, in turn, be due to the "canalizing" influence of modifier loci (Waddington 1975). Second, strong "coadapted" gene complexes or epistatic interactions among loci may ensure that nearly all variation that arises is severely deleterious. To use Wright's metaphor, species are on peaks in a rugged fitness landscape from which it is difficult to shift them (Wright 1977). Third, if there is variation, it is bound up in strong pleiotropic relations among traits that, cumulatively, prevent selection on any one component of the organisms' phenotype (Coddington 1990; Baum and Larson 1991; Harvey and Pagel 1991).

How plausible is each of these explanations for phylogenetic conservatism? There are at least a few examples of phylogenetically conserved traits for which some evidence indicates that stabilizing selection does not play a large role in their maintenance. Very few dipterans, for instance, show any form of directional asymmetry. Repeated laboratory selection experiments on Drosophila, for example, have failed to detect any heritable variation for directional asymmetry (see, e.g., Tuinstra et al. 1990). On the other hand, a possible example of a trait phylogenetically conserved by stabilizing selection is provided by natural experiments in clades with cave-dwelling taxa. The presence of eyes is uniform in the fish family Characidae, except for populations of the blind cave fish of Mexico, Astyanax mexicanus, that were isolated from their sighted surface dwelling relatives about $10,000 \mathrm{yr}$ ago. It is unclear whether this example of recessive evolution is due to mutation pressure, direct selection for eye loss, or selection on some pleiotropic effect (Culver 1982), but it is a striking demonstration of the presence of genetic variation for a trait that is ancient, conserved at high taxonomic levels, and functional.

Directional asymmetry in Drosophila is remarkable in possessing little selectable variation; few other traits subjected to selection in the laboratory fail to respond (Maynard Smith 1989). Just as a lack of genetic variation cannot be generally held responsible for stasis in the fossil record (Charlesworth et al. 1982), 
so too it cannot explain stasis in phylogenies. We suspect that striking cases of phylogenetic conservatism are the product of complex patterns of epistasis and pleiotropy, manifest as strong genetic correlations among traits and complex patterns of correlated selection. In morphological traits, such patterns arise as a consequence of development (Arnold 1992; Atchley et al. 1992). Sinervo and Licht (1991) experimentally demonstrated complex patterns of selection in lifehistory traits. They showed that the pelvic girdle dimensions of a lizard, Uta stansburiana, impose an upper selective limit to egg size and, since clutch size and egg size trade off phenotypically, a lower selective limit to clutch size. Although the question remains open as to what, in turn, limits pelvic girdle dimensions, it is easy to see how selection for, say, locomotor performance, could have ramifying effects on life-history traits. Possibly such patterns of selection are responsible for striking cases of conserved clutch sizes such as that of the Gekkonid lizards, 600 species of which invariably lay two eggs (Stearns 1984), and those of the genus Anolis (anoles), which invariably lay but one (Sinervo and Licht 1991). The important point is that there is no obvious way of distinguishing among the several notions of "constraint" without measuring current and historical patterns of selection, genetic variation, and genetic covariation.

\section{Unwarranted Inferences of Adaptation and Constraint from Correlations among Characters}

As discussed earlier, there are also at least three distinct evolutionary genetic explanations for interspecific correlations among traits. We have called these the pleiotropy, epigenetics, and correlated selection hypotheses. While the notion of "epigenetics" (sensu Atchley and Hall 1991) is relatively new and has not been used extensively in the comparative literature (but see Atchley et al. 1992), pleiotropy and correlated selection have long coexisted in the comparative literature as explanations for interspecific associations among traits. Curiously, they are rarely encountered as alternative explanations for any single pattern. On the one hand, motivated by particular notions of the physiological or developmental relations among traits, comparative biologists have often searched the phylogenetic record for the influence of pleiotropy writ large. Traits related to body size and life history have often been subject to this sort of interpretation. On the other hand, a glance at any current text on behavioral or evolutionary ecology will reveal numerous adaptive hypotheses concerning two or more traits in which no mention is made of the genetic relations that the traits may bear to each other. In this section we counterpose alternative evolutionary explanations for two of the best-documented and best-understood comparative patterns available, namely brain-body allometry and the effects of costs of reproduction on life history.

Brain-body allometry. - The scaling of brain and body size over orders of placental mammals can be described as a power function (Gould 1977; Lande 1979):

$$
\log (\text { Brain })=\log (k)+b \log (\text { Body }), \text { where } 0.67<b<0.75 \text {. }
$$

The allometric coefficient, $b$, which is the slope of the linear relationship between log-transformed brain and body size, is sometimes smaller at lower taxonomic categories than at higher (e.g., the slope best describing this relationship 
may be shallower among or within genera, than among or within orders; Lande 1979; Pagel and Harvey 1989). The interspecific relationship between brain and body size and its variation among taxa or taxonomic levels are unusual among such patterns in that something is known of the genetic relationships between these traits (Roderick et al. 1976; Atchley et al. 1984). Furthermore, this pattern has been the subject of a number of explicit quantitative genetic models-some comparable to those discussed earlier in this article (Lande 1979; Riska and Atchley 1985; Riska 1989) — that have attempted to explain variation in the allometric coefficient among taxa.

Using quantitative genetic data from laboratory mice, Lande (1979) argued that relatively small allometric coefficients $(b=0.37)$ could be explained by selection for body size alone. Larger allometric coefficients $(b=0.67)$, on the other hand, were closer to those that might be expected from selection for brain size alone. Riska and Atchley (1985), observing that the genetic correlation between body size and adult brain size in mice changes over the course of ontogeny, modified Lande's hypothesis. They argued that evolutionary changes in brain size could be explained by selection for body size alone and that differences in allometric coefficients were attributable to selection having acted on different components of body growth. Others have suggested that the evolution of mammalian neonatal brain size of mammals is constrained by maternal metabolic rate (Martin 1981) or that it has evolved with gestation length, litter size, and other life-history traits (Pagel and Harvey 1988).

The history of brain-body allometry studies illustrates at least three of the problems that we believe to be inherent to the comparative method. The first is that of confounding selection pressures. Insofar as mammalian body size is phenotypically and genetically correlated with innumerable other traits-possibly including fitness components such as fecundity and mating success-the claim that selection on adult body size rather than brain size is responsible for the pattern of allometry within a certain taxon is tantamount simply to choosing one of a very large number of possibly equally correlated variables, and the same is true for any claim concerning selection on brain size.

The second problem lies in the confounding of the causal influence of selection with that of genetic correlations. In the case of brain-body allometry, this error manifests itself as the notion that allometric coefficients can reflect the degree to which one trait constrains the evolution of another. For example, while Martin (1981) and Pagel and Harvey (1989) disagree as to whether neonatal brain size and maternal body weight scale isometrically, both apparently feel that the value of $b$ can be used as evidence for or against constraint hypotheses. This is a use of allometric coefficients that ignores the salutary lesson of Lande's (1979) article, namely that the constraints on evolution are not allometric relations but rather the particular genetic relations among traits and the historical selection forces that give rise to allometric relations. Since neither genetic relations nor historical selection forces are recoverable from allometric relations without external knowledge of at least one or the other (Riska 1989), it follows that the causal effect of the evolution of one trait on another can be neither confirmed nor falsified by the finding of a particular allometric coefficient. 
Finally, we suspect that the empirical quantitative genetic foundation of brainbody allometric models is less than firm. The estimates of genetic correlations between brain and body size used by Lande (1979) and Riska and Atchley (1985) come from populations of laboratory mice. Although perhaps better than nothing at all, such data probably tell us little about the genetic structure of mammalian populations in general. This observation is because genetic correlations change with environment (Service and Rose 1985) and population structure, particularly since laboratory mouse populations are at least somewhat inbred (see, e.g., Roderick et al. 1976) and inbreeding is known to alter genetic correlations (Rose 1984a). In addition, there is the problem of the phylogenetic generalization from mice to other species of mammal. We know of no evidence bearing on the constancy of genetic correlations among brain size, body size, and growth components over higher mammalian taxa. If any of these factors have influenced the patterns of genetic variance and covariance for brain and body size within the Mammalia, then there is virtually no limit to the combinations of selective forces and genetic parameters that could account equally well for the observed patterns (Riska 1989).

Costs of reproduction and the evolution of life history.-Williams (1966a, $1966 b$ ) suggested that effort expended on reproduction early in life would cause a diminution of residual reproductive value. In other words, early reproduction has a cost, one that is paid out of subsequent reproduction (e.g., by the reduction of later survival). Williams also suggested that such a cost of reproduction might be reflected in a negative correlation over species between some index of reproductive effort and the residual reproductive value (of which survival is the most easily measured component; Williams 1966a, 1966b). Many studies, using data from natural populations, have shown a negative relationship across species between reproductive effort and survival (Tinkle 1969; Saether 1988; Harvey et al. 1990; Promislow and Harvey 1990). Are comparative studies of this sort adequate tests of Williams's theory? If we understand the theory to be a statement about evolutionary mechanism, specifically, the presence of negative genetic correlations among early and late reproductive output, then, for at least three reasons, we believe they are not.

First, in common with comparative studies of brain-body allometry, these studies inevitably confound the effects of correlated selection on the distribution of traits among species with those of genetic correlations. Are negative correlations among life-history traits across species the product of a true cost of reproduction (an antagonistic pleiotropy), or are they due to simultaneous direct selection on separate life-history traits? Short of the quantitative genetic analysis of individual species, there seems to be no way to tell.

Second, the effects of environmental differences among species on correlations among life-history traits are often confounded with those due to genetic differences (see, e.g., Harvey and Zammuto 1985; Saether 1988; Promislow and Harvey 1990). This problem is especially acute in studies of life-history traits, which are often very sensitive to environmental variation and often respond in opposite ways to environmental variation (i.e., they have negative environmental covariances). For example, if individuals adjust their fecundity in response to mortality 
such that population sizes are roughly constant over time, then negative correlations between components of fecundity and survival among populations and higher taxa will be inevitable and will perniciously mimic the effects of a genetic trade-off (Sutherland et al. 1986; Gustafsson and Sutherland 1988; Lindén and Møller 1989; Harvey and Keymer 1991; Partridge and Sibly 1991). Ecological effects may be removed by comparing laboratory or zoo populations (see, e.g., Schnebel and Grossfield 1988; Harvey et al. 1990). However, if some species are preadapted to the laboratory while others are not, trade-offs that obtain within each species may be obscured when they are compared. This result is possibly why trade-offs between early fecundity and longevity have not been found when Drosophila species and semispecies are compared in the laboratory (Schnebel and Grossfield 1988), even though such trade-offs do exist within outbred laboratory populations of Drosophila melanogaster (Rose and Charlesworth 1981a, 1981b; Rose 1984b).

Third, evolution of genetic background may obscure differences in the costs of reproduction paid by various species. For example, evolutionary changes in body size should have widespread effects on organismal physiology, including many aspects of energetic acquisition. For this reason, some workers have advocated testing for interspecific costs of reproduction only once the effects of body size have been removed (Promislow and Harvey 1990; Blackburn 1991; Harvey and Pagel 1991). Typically, when the effects of body size have been removed, negative interspecific correlations are found among some life-history traits but not among others (see, e.g., Promislow and Harvey 1990). Such results tell little, however, about which life-history traits are influenced by costs of reproduction and which are not. Body size is but the most obvious of a multitude of unknown physiological traits on which selection could act so as to obscure trade-offs, other traits that cannot be easily measured or statistically "removed." The existence of such traits means that the interspecific variation that remains after the effects of body size have been removed are in no sense necessarily a true representation of either the functional or genetic relationships among traits.

Taken together, the factors that we have discussed-correlated selection, covariation of traits due to environment variation, differences among species in their phenotypic responses to environmental variation, and the effects of genetic background-can give rise to negative interspecific correlations among lifehistory traits when costs of reproduction are absent and positive correlations when they exist. Unlike Harvey and Keymer (1991), however, we find it difficult to see how the collection of more comparative data, or application of more sophisticated statistical methodology, could mitigate these problems.

THE USES OF THE COMPARATIVE METHOD

Comparison of the average characteristics of species, their performances, and environments reveals little about adaptation and evolutionary constraint because the forces that mold evolution-selection, drift, mutation, and migration, and the substrate of genetic variation and covariation on which they act-are complex and hopelessly confounded in the way in which they affect the relative attributes 
of different taxa. Yet it should not be thought that measuring genetic correlations and selection coefficients in any one population will reveal much about either its adaptedness to its environment or lack thereof. A population is the product of its history: measurement of its current parameters probably tells less about its past than its immediate future.

However, we believe that the methods of comparative biology and evolutionary genetics might be usefully combined. Specifically, patterns of selection, genetic variation, covariation, and interaction might be measured for several species belonging to a single monophyletic group; the historical influence of these various forces might then be inferred with the aid of standard phylogenetic techniques such as optimization (see, e.g., Brooks and McLennan 1991). For example, to test the notion that two traits have been constrained to evolve together in some clade, we suggest that their genetic correlation should be measured in each of the terminal taxa of that clade. This approach is not an infallible method; it is no better than the quantitative genetic and phylogenetic methods on which it is based. But we would be far surer of the historical existence of a constraint so inferred than of one based only on a priori notions of function or development. The selection history of a trait in a clade could be inferred in an analogous fashion instead of by the mere assertion that a clade has made a historical transition in lifestyle or habitat.

Although there are a few comparative studies of genetic variance and covariance matrices (Arnold 1981; Lofsvold 1986; Atchley et al. 1992), phylogenetic analyses of the evolution of genetic parameters hardly exist. One exception is that of Cohan and Hoffmann (1989), who compared the pattern of correlated responses to selection for knock-down ethanol resistance in species belonging to the melanogaster subgroup and obscura subgroup of Drosophila. The most elegant example that we know of a phylogenetic study of selection is Basolo's (1990) study of female preference for male ornamentation in the genus Xiphophorus. The approach advocated here, although using experimental data, remains part of comparative methodology and would take advantage of all of the recent conceptual and technical advances of that field.

There are also situations in which genuine experimental manipulations can be done to reveal the basis of a comparative pattern. Reznick and colleagues (Reznick and Endler 1982; Reznick and Bryga 1987; Reznick 1989) have shown how among-population differences in the life-history attributes of Trinidadian guppies are attributable to varying patterns of predation. This conclusion was reached through a combination of quantitative genetic studies, replicated ecological comparisons, and, most strikingly, experiments in which the selective pressures on natural populations were altered and their subsequent evolution observed. Such experiments may well be the most powerful way in which adaptation can be studied.

Unlike Gould and Lewontin (1979), we do not hypothesize that many traits are not adaptive. Rather, we are making the case that the adaptive (or nonadaptive) nature of traits cannot be determined from most comparative data. If, as we suggest, historical study of the evolutionary process is subject to strict limitations, then the various terms proposed to describe such processes or characters are also 
subject to greatly restricted use. These include the terms adaptation, exaptation, disaptation, or aptation (Gould and Vrba 1982; Baum and Larson 1991), all of which imply direct and specific knowledge of selection forces in the past and the pattern of genetic correlation among characters. A further implication of our thesis is that the promise of phylogenetic analyses of congruence among traits, performance variables, and environmental characters to demonstrate adaptations will be unfulfilled.

We do recognize the rather severe limitations that our prescriptions place on retrospective studies of adaptation and evolutionary constraint. But we consider that such limitations are better seen clearly than obscured by false hopes about the ability of comparative studies to resolve the processes of evolution. Indeed, process is not all that is interesting in evolution; we can still learn a great deal about the nature of organismal diversity by describing and analyzing patterns of character evolution alone (Eldredge and Cracraft 1980; Lauder 1981; Wiley 1981; Lauder and Liem 1989; Brooks and McLennan 1991). Such analyses will be all the more robust for lacking unsupported assumptions, unwarranted inferences, and untestable hypotheses about the history of evolutionary mechanisms.

\section{ACKNOWLEDGMENTS}

We thank A. F. Bennett, J. Cracraft, S. A. Frank, A. Gibbs, M. Matos, and L. D. Mueller for their insightful comments on the manuscript. This work was supported in part by U.S. Public Health Service grants AG0-6346 and AG0-9970 to M.R.R. and National Science Foundation grant IBN 91-19502 to G.V.L.

\section{LITERATURE CITED}

Antonovics, J., and P. H. van Tienderen. 1991. Ontoecogenophyloconstraints? The chaos of constraints terminology. Trends in Ecology \& Evolution 6:166.

Arnold, S. J. 1981. Behavioral variation in natural populations. I. Phenotypic, genetic and environmental responses to prey in the garter snake, Thamnophis elegans. Evolution 35:489-509. 1992. Constraints on phenotypic evolution. American Naturalist 140(suppl.):85S-107S.

Atchley, W. R., and B. K. Hall. 1991. A model for development and evolution of complex morphological structures. Biological Reviews of the Cambridge Philosophical Society 66:101-157.

Atchley, W. R., B. Riska, L. A. Kohn, A. A. Plummer, and J. J. Rutledge. 1984. A quantitative genetic analysis of brain and body size associations, their origin and ontogeny: data from mice. Evolution 38:1165-1179.

Atchley, W. R., D. E. Cowley, C. Vogel, and T. McLellan. 1992. Evolutionary divergence, shape change, and genetic correlation structure in the rodent mandible. Systematic Biology 41: 196-221.

Barton, N. H., and M. Turelli. 1989. Evolutionary quantitative genetics: how little do we know? Annual Reviews of Genetics 23:337-370.

Basolo, A. 1990. Female preference predates the evolution of the sword in swordtail fish. Science (Washington, D.C.) 250:808-810.

Baum, D. A., and A. Larson. 1991. Adaptation reviewed: a phylogenetic methodology for studying character macroevolution. Systematic Zoology 40:1-18.

Berven, K. A., and D. E. Gill. 1982. The genetic basis of altitudinal variation in the wood frog, Rana sylvatica. II. An experimental analysis of larval traits. Oecologia (Berlin) 52:360-369.

Blackburn, T. M. 1991. The interspecific relationship between egg size and clutch size in wildfowl. Auk 108:209-211. 
Bock, W. 1977. Adaptation and the comparative method. Pages 57-82 in M. Hecht, ed. Major patterns in vertebrate evolution. Plenum, New York.

Brandon, R. H. 1990. Adaptation and environment. Princeton University Press, Princeton, N.J.

Brooks, D. R., and D. A. McLennan. 1991. Phylogeny, ecology and behavior. University of Chicago Press, Chicago.

Charlesworth, B., R. Lande, and M. Slatkin. 1982. A neo-Darwinian commentary on macroevolution. Evolution 36:474-498.

Clark, A. G., and M. W. Feldman. 1981. The estimation of epistasis in components of fitness in experimental populations of Drosophila melanogaster. II. Assessment of meiotic drive, viability, fecundity and sexual selection. Heredity 46:347-377.

Clark, A. G., M. W. Feldman, and F. B. Christiansen. 1981. The estimation of epistasis in components of fitness in experimental populations of Drosophila melanogaster. I. A two stage maximum likelihood model. Heredity 46:321-346.

Clarke, G. M., and J. A. McKenzie. 1987. Developmental stability of insecticide resistant phenotypes in blowfly: a result of canalizing natural selection. Nature (London) 325:345-346.

Clutton-Brock, T. H., and P. H. Harvey. 1979. Comparison and adaptation. Proceedings of the Royal Society of London B, Biological Sciences 205:547-565.

Coddington, J. A. 1988. Cladistic tests of adaptational hypotheses. Cladistics 4:3-22.

- 1990. Bridges between evolutionary pattern and process. Cladistics 6:379-386.

Cody, M., and J. Diamond, eds. 1975. Ecology and evolution of communities. Belknap, Cambridge, Mass.

Cohan, F. M., and A. A. Hoffmann. 1989. Uniform selection as a diversifying force in evolution: evidence from Drosophila. American Naturalist 134:613-637.

Cowley, D. E., and W. R. Atchley. 1990. Developmental and quantitative genetics of correlation structure among body parts of Drosophila melanogaster. American Naturalist 135:242-268.

Cracraft, J. 1981. The use of functional and adaptive criteria in phylogenetic systematics. American Zoologist 21:21-36.

Crow, J. 1987. Population genetics history: a personal view. Annual Review of Genetics 21:1-22.

Culver, D. C. 1982. Cave life. Harvard University Press, Cambridge, Mass.

Darwin, C. 1859. On the origin of species. (Facsimile of the first edition, 1967.) Atheneum, New York.

1871. The descent of man and selection in relation to sex. Pt. 2. (Facsimile of the first edition, 1989.) Pickering, London.

de Jong, G. 1990. Genotype-by-environment interaction and the genetic covariance between environments: multilocus genetics. Genetica 81:171-177.

Eldredge, N., and J. Cracraft. 1980. Phylogenetic patterns and the evolutionary process. Columbia University Press, New York.

Endler, J. 1986. Natural selection in the wild. Princeton University Press, Princeton, N.J.

Felsenstein, J. 1985. Phylogenies and the comparative method. American Naturalist 125:1-15.

Futuyma, D. 1986. Evolutionary biology. 2d ed. Sinauer, Sunderland, Mass.

Gould, S. J. 1977. Ontogeny and phylogeny. Belknap, Cambridge, Mass.

Gould, S. J., and R. C. Lewontin. 1979. The spandrels of San Marco and the Panglossian paradigm: a critique of the adaptationist programme. Proceedings of the Royal Society of London B, Biological Sciences 205:581-598.

Gould, S. J., and E. S. Vrba. 1982. Exaptation-a missing term in the science of form. Paleobiology $8: 4-15$.

Graves, J. L., E. C. Toolson, C. Jeong, L. N. Vu, and M. R. Rose. 1992. Desiccation, flight, glycogen, and postponed senescence in Drosophila melanogaster. Physiological Zoology 65:268-286.

Greene, H. W. 1986. Diet and arboreality in the Emerald Monitor, Varanus prasinus, with comments on the study of adaptation. Fieldiana Zoology 31:1-12.

Gustafsson, L., and W. J. Sutherland. 1988. The costs of reproduction in the collard flycatcher Ficedula albicollis. Nature (London) 335:813-815.

Harvey, P. H., and A. E. Keymer. 1991. Comparing life histories using phylogenies. Philosophical Transactions of the Royal Society of London B, Biological Sciences 332:31-39.

Harvey, P. H., and M. D. Pagel. 1991. The comparative method in evolutionary biology. Oxford University Press, New York. 
Harvey, P. H., and T. M. Zammuto. 1985. Patterns of mortality and age at first reproduction in natural populations of mammals. Nature (London) 315:319-320.

Harvey, P. H., A. F. Read, and D. E. Promislow. 1990. Life history variation in placental mammals: unifying the data with theory. Oxford Surveys in Evolutionary Biology 6:13-31.

Hoffmann, A. A., and P. A. Parsons. 1991. Evolutionary genetics and environmental stress. Oxford University Press, New York.

Houle, D. 1991. Genetic covariance of fitness correlates: what genetic correlations are made of and why it matters. Evolution 45:630-645.

Jinks, J. L., J. M. Perkins, and H. S. Pooni. 1973. The incidence of epistasis in normal and extreme environments. Heredity 31:263-269.

Keightley, P. 1989. Models of quantitative variation of flux in metabolic pathways. Genetics 121: 869-876.

Kimura, M. 1983. The neutral theory of molecular evolution. Cambridge University Press, Cambridge.

King, J. C. 1955. Evidence for the integration of the gene pool from studies of DDT resistance in Drosophila. Cold Spring Harbour Symposia on Quantitative Biology 20:311-317.

Kohn, L. A., and W. A. Atchley. 1988. How similar are genetic correlation structures? data from rats and mice. Evolution 42:467-481.

Kreitman, M. 1983. Nucleotide polymorphism at the alcohol dehydrogenase locus of Drosophila melanogaster. Nature (London) 304:412-417.

Lande, R. 1979. Quantitative genetic analysis of multivariate evolution, applied to brain: body allometry. Evolution 33:402-416.

Larson, A. 1984. Neontological inferences of evolutionary pattern and process in the salamander family Plethodontidae. Evolutionary Biology 17:119-217.

Larson, A., D. B. Wake, L. R. Maxson, and R. Highton. 1981. A molecular phylogenetic perspective on the origins of morphological novelties in the salamanders of the tribe Plethodontini (Amphibia, Plethodontidae). Evolution 35:405-422.

Lauder, G. V. 1981. Form and function: structural analysis in evolutionary morphology. Paleobiology 7:430-442.

Lauder, G. V., and K. F. Liem. 1989. The role of historical factors in the evolution of complex organismal functions. Pages 63-78 in D. B. Wake and G. Roth, eds. Complex organismal functions: integration and evolution in vertebrates. Wiley, Chichester.

Lenski, R. 1988a. Experimental studies of pleiotropy and epistasis in Escherichia coli. I. Variation in competitive fitness among mutants resistant to virus T4. Evolution 42:425-432.

$1988 b$. Experimental studies of pleiotropy and epistasis in Escherichia coli. II. Compensation for maladaptive effects associated with resistance to virus T4. Evolution 42:433440.

Lindén, M., and A. P. Møller. 1989. Cost of reproduction and covariation of life-history traits in birds. Trends in Ecology \& Evolution 4:367-371.

Lofsvold, D. 1986. Quantitative genetics of morphological differentiation in Peromyscus. I. Tests of the homogeneity of genetic covariance structure among species and subspecies. Evolution 40:559-573.

Luckinbill, L. S., J. L. Graves, A. Tomkiw, and O. Sowirka. 1988. A qualitative analysis of life history characters in Drosophila melanogaster. Evolutionary Ecology 3:31-39.

Luke, C. 1986. Convergent evolution of lizard toe fringes. Biological Journal of the Linnean Society 27:1-16.

Martin, R. D. 1981. Relative brain size and basal metabolic rate in terrestrial vertebrates. Nature (London) 293:57-59.

Maynard Smith, J. 1989. Evolutionary genetics. Oxford University Press, New York.

Mayr, E. 1983. How to carry out the adaptationist program? American Naturalist 121:324-334.

McKenzie, J. A., M. J. Whitten, and M. A. Adena. 1982. The effect of genetic background on the fitness of diazinon resistance genotypes of the Australian sheep blowfly Lucilia cuprina. Heredity 49:1-9.

McLennan, D. A., D. R. Brooks, and J. D. McPhail. 1988. The benefit of communication between comparative ethology and phylogenetic systematics: a case study using gasterosteid fishes. Canadian Journal of Zoology 66:2177-2190.

Mishler, B. D. 1988. Reproductive ecology of bryophytes. Pages 285-306 in J. Lovett Doust and 
L. Lovett Doust, eds. Plant reproductive ecology: patterns and strategies. Oxford University Press, New York.

Pagel, M. D., and P. H. Harvey. 1988. How mammals produce large-brained offspring. Evolution 42:948-957.

1989. Taxonomic differences in the scaling of brain on body weight among mammals. Science (Washington, D.C.) 244:1589-1593.

Partridge, L., and R. Sibly. 1991. Constraints in the evolution of life histories. Philosophical Transactions of the Royal Society of London B, Biological Sciences 332:3-13.

Promislow, D. E. L., and P. H. Harvey. 1990. Living fast and dying young: a comparative analysis of life-history variation among mammals. Journal of Zoology (London) 220:417-437.

Reznick, D. N. 1989. Life-history evolution in guppies. II. Repeatability of field observations and the effects of season on life histories. Evolution 43:1285-1297.

Reznick, D. N., and H. Bryga. 1987. Life-history evolution in guppies (Poecilia reticulata). I. Phenotypic and genetic changes in an introduction experiment. Evolution 41:1370-1385.

Reznick, D. N., and J. A. Endler. 1982. The impact of predation on life history evolution in Trinidadian guppies (Poecilia reticulata). Evolution 36:160-177.

Ridley, M. 1992. Darwin sound on comparative method. Trends in Ecology \& Evolution 7:37.

Riska, B. 1989. Composite traits, selection response, and evolution. Evolution 43:1172-1191.

Riska, B., and W. R. Atchley. 1985. Genetics of growth predict patterns of brain-size evolution. Science (Washington, D.C.) 229:668-671.

Roderick, T. H., R. E. Wimmer, and C. C. Wimmer. 1976. Genetic manipulation of neuroanatomical traits. Pages 143-178 in L. Petrovich and J. L. McGaugh, eds. Knowing, thinking, and believing. Plenum, New York.

Rose, M. R. 1982. Antagonistic pleiotropy, dominance, and genetic variation. Heredity 48:63-78.

. 1983. Further models of selection with antagonistic pleiotropy. Pages 47-53 in H. I. Freeman and C. Strobeck, eds. Population biology. Springer, Berlin.

1984a. Genetic covariation in Drosophila life history: untangling the data. American Naturalist 123:565-569.

1984b. Laboratory evolution of postponed senescence in Drosophila melanogaster. Evolution 38:1004-1010.

Rose, M. R., and B. Charlesworth. 1981a. Genetics of life-history in Drosophila melanogaster. I. Sib analysis of adult females. Genetics 97:173-186.

. 1981b. Genetics of life-history in Drosophila melanogaster. II. Exploratory selection experiments. Genetics 97:187-196.

Saether, B.-E. 1988. Pattern of covariation between life-history traits of European birds. Nature (London) 331:616-617.

Schlichting, C. D., and D. A. Levin. 1986. Phenotypic plasticity: an evolving plant character. Biological Journal of the Linnean Society 29:37-47.

Schnebel, E. M., and J. Grossfield. 1988. Antagonistic pleiotropy: an interspecific Drosophila comparison. Evolution 42:306-311.

Seager, R. D., and F. J. Ayala. 1982. Chromosome interactions in Drosophila melanogaster. I. Viability studies. Genetics 102:467-483.

Service, P. M. 1987. Physiological mechanisms of increased stress resistance in Drosophila melanogaster selected for postponed senescence. Physiological Zoology 60:321-326.

1993. Laboratory evolution of longevity and reproductive fitness components in male fruit flies: mating ability. Evolution 47:387-399.

Service, P. M., and R. M. Rose. 1985. Genetic co-variation among life-history components: the effects of novel environments. Evolution 39:943-945.

Service, P. M., E. W. Hutchinson, M. D. MacKinley, and M. R. Rose. 1985. Resistance to environmental stress in Drosophila melanogaster selected for postponed senescence. Physiological Zoology 58:380-389.

Service, P. M., E. W. Hutchinson, and M. R. Rose. 1988. Multiple genetic mechanisms for the evolution of senescence in Drosophila melanogaster. Evolution 39:708-716.

Sheridan, A. K., and J. S. F. Barker. 1974. Two-trait selection and genetic correlation. II. Changes in the genetic correlation during two-trait selection. Australian Journal of Biological Sciences 27:75-88. 
Simpson, G. G. 1953. The major features of evolution. Columbia University Press, New York.

Sinervo, B., and P. Licht. 1991. Proximate constraints on the evolution of egg size, number and total clutch mass in lizards. Science (Washington, D.C.) 252:1300-1302.

Slatkin, M., and S. A. Frank. 1990. The quantitative genetic consequences of pleiotropy under stabilizing and directional selection. Genetics 125:207-213.

Sober, E. 1984. The nature of selection: evolutionary theory in philosophical focus. MIT Press, Cambridge, Mass.

Stearns, S. 1984. The tension between adaptation and constraint in the evolution of reproductive patterns. Advances in Invertebrate Reproduction 3:387-398.

Stearns, S. C., G. de Jong, and G. Newman. 1991. The effects of phenotypic plasticity on genetic correlations. Trends in Ecology \& Evolution 6:122-126.

Sutherland, W. J., A. Grafen, and P. H. Harvey. 1986. Life history correlations and demography. Nature (London) 320:88.

Tinkle, D. W. 1969. The concept of reproductive effort and its relation to the evolution of life histories of lizards. American Naturalist 102:501-516.

Traub, R. 1980. Some adaptive modifications in fleas. Pages 33-67 in R. Traub and H. Starcke, eds. Proceedings of the International Conference on Fleas, Ashton Wold, U.K., June 1977. Balkema, Rotterdam.

Trivers, R. 1985. Social evolution. Cummings, Menlo Park, Calif.

Tuinstra, E. J., G. de Jong, and W. Scharloo. 1990. Lack of response to family selection for directional asymmetry in Drosophila melanogaster: left and right are not distinguished during development. Proceedings of the Royal Society of London B, Biological Sciences 241:146-152.

Turelli, M. 1988. Phenotypic evolution, constant covariances, and the maintenance of additive variation. Evolution 42:1342-1347.

Van Valen, L. 1971. Adaptive zones and the orders of mammals. Evolution 25:420-428.

Waddington, C. H. 1975. The evolution of an evolutionist. Cornell University Press, Ithaca, N.Y.

Wake, D. B., and A. Larson. 1987. Multidimensional analysis of an evolving lineage. Science (Washington, D.C.) 238:42-48.

Wiley, E. O. 1981. Phylogenetics: the theory and practice of phylogenetic systematics. Wiley Interscience, New York.

Wilkinson, G., K. Fowler, and L. Partridge. 1990. Resistance of genetic correlation structure to directional selection in Drosophila melanogaster. Evolution 44:1990-2003.

Williams, G. C. 1966a. Adaptation and natural selection. Princeton University Press, Princeton, N.J. . 1966 b. Natural selection, the costs of reproduction and a refinement of Lack's principle. American Naturalist 100:687-690.

Wright, S. 1977. Evolution and the genetics of populations. University of Chicago Press, Chicago. 